\title{
TOWARDS A QUANTITATIVE MODEL OF EPIDEMICS DURING CONFLICTS
}

Soumya Banerjee*

University of Oxford, Mathematical Institute

Oxford, United Kingdom

DOI: $10.7906 /$ indecs.17.3.16

Received: 27 April 2019.

Regular article

Accepted: 10 September 2019.

\begin{abstract}
Epidemics may contribute to and arise as a result of conflict. The effects of conflict on infectious diseases are complex. There have been counter-intuitive observations of both increase and decrease in disease outbreaks during and after conflicts. However there is no unified mathematical model that explains all these observations. There is an urgent need for a quantitative framework for modelling conflicts and epidemics. The article introduces a set of mathematical models to understand the role of conflicts in epidemics. The corresponding mathematical framework has the potential to explain the counter-intuitive observations and the complex role of human conflicts in epidemics. This work suggests that aid and peacekeeping organizations should take an integrated approach that combines public health measures, socio-economic development, and peacekeeping in conflict zones.

This approach exemplifies the role of non-linear thinking in complex systems like human societies. The work presented should be looked upon as a first step towards a quantitative model of disease spread in conflicts.
\end{abstract}

\section{KEY WORDS}

simulation models, epidemics, conflicts

\section{CLASSIFICATION}

JEL: $\quad$ C65

PACS: $87.19 . \mathrm{Xx}, 89.90 .+\mathrm{n}$ 


\section{INTRODUCTION}

Epidemics and conflicts are closely connected. Epidemics may both contribute to conflict and also arise as a result of violence in human societies. The effects of conflict on infectious diseases are multi-faceted and complex. There have been counter-intuitive observations of both increase and decrease in disease outbreaks during and after conflicts [1]. For example, epidemics have been observed to be both initiated and diminished by conflicts. Paradoxically, epidemics have been observed to rebound, even after conflicts have ended. However there is no unified quantitative model that explains all these counter-intuitive observations.

There is an urgent need for a quantitative framework for modelling conflicts and epidemics. The recent appearance of emerging pathogens like Zika and Ebola virus in conflict-prone regions highlights the need for a quantitative framework that combines the effects of both disease spread and conflicts.

Such models can be a first step towards shaping public health policy, spreading public awareness and may also be a tool for public health professionals in conflict zones.

Quantitative techniques like these may also help predict possible emerging hotspots for emerging diseases [2]. Models of joint epidemic and conflict risk may be of considerable interest to future humanitarian and peacekeeping missions.

\section{MODELS}

We start with a basic susceptible-infected-recovered (SIR) model. The density of susceptible people who are healthy but can be infected by a pathogen is denoted by $S$. The density of people who are infected is represented by the compartment $I$. The interaction between infected and susceptible causes more infections which is represented by the mass action term $-\beta I S$ (the rate at which susceptibles become infected). This shows up as influx term in the infected compartment $(+\beta I S)$. The density of people who recover from infections is represented by $R$. This is composed of an influx from the infected population at a rate of $v I$. The model is shown below.

$$
\begin{gathered}
\frac{\mathrm{d} S}{\mathrm{~d} t}=-\beta I S, \\
\frac{\mathrm{d} I}{\mathrm{~d} t}=\beta I S-v I, \\
\frac{\mathrm{d} R}{\mathrm{~d} t}=v I .
\end{gathered}
$$

We assume in this simple model that those who recover never become infected again. Additionally, we neglect birth and death processes in this simple model. All these assumptions can be relaxed in more complex models. A simulation of this simple model is shown further in Figure 1.

The basic reproductive number $(R$,$) is the expected number of new infections produced by a$ single infected individual over the individual's productively infected lifespan (in a completely susceptible population). It is given by the following quantity

$$
R_{0}=\frac{\beta \mathrm{S}}{\mathrm{v}} \text {. }
$$

This can be derived by observing that the infection can be sustained if the rate of change of infected individuals $(I)$ is greater than 0 :

$$
\frac{\mathrm{d} I}{\mathrm{~d} t}=\beta I S-v I>0 \Rightarrow \beta I S>v I .
$$

This finally gives us the following relationship

$$
R_{0}=\frac{\beta S}{v}>1
$$




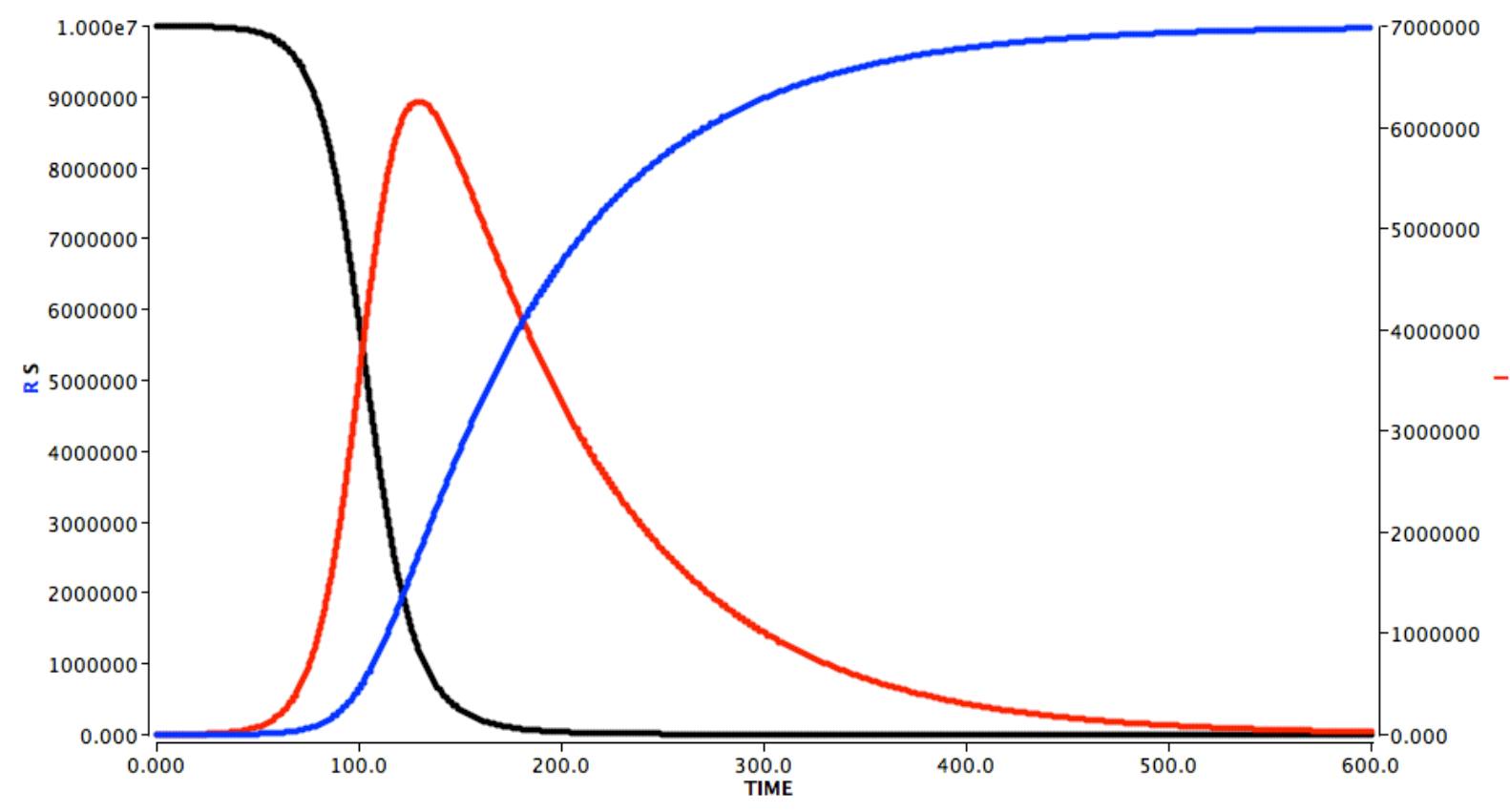

Figure 1. A simulation of the basic SIR model showing how the density of infected people ( $I$, in red) rises and then decreases. The density of susceptible people ( $S$, in black) declines causing the infection to start declining. The density of people who recover $(R$, in blue) increases throughout the process.

\section{METHODS}

The dynamical models were implemented in Berkeley Madonna [3] and have been made available online [4].

\section{RESULTS}

\section{COUPLED MODELS OF DISEASE AND CONFLICT}

We introduce the following model of two populations in conflict with each other. $S_{1}$ and $S_{2}$ refer to susceptible populations of two nations or communities within a nation (combines both civilians and combatants). The first term in each of these compartments simulates removal due to infection. The second term models influx or efflux of people: this could be due to refugees fleeing from one country to another or the invasion of an army. This is modelled as a piecewise linear function $(X)$.

$$
\begin{aligned}
& \frac{\mathrm{d} S_{1}}{\mathrm{~d} t}=-\beta I S_{1}+\alpha_{1} X, \\
& \frac{\mathrm{d} S_{2}}{\mathrm{~d} t}=-\beta I S_{2}+\alpha_{2} X, \\
& \frac{\mathrm{d} I}{\mathrm{~d} t}=\beta I S_{1}+\beta I S_{2}-v I, \\
& \frac{\mathrm{d} R}{\mathrm{~d} t}=v I . \\
& X=\left\{\begin{array}{cc}
0, & t<t_{i}, \\
\delta \cdot\left(t-t_{i}\right), & t \geq t_{i} .
\end{array}\right.
\end{aligned}
$$

We note that some of these parameters might be correlated, e.g. increased migration might breakdown already strained medical services (parameter $v$ in the SIR model). We look at this in a later model. 


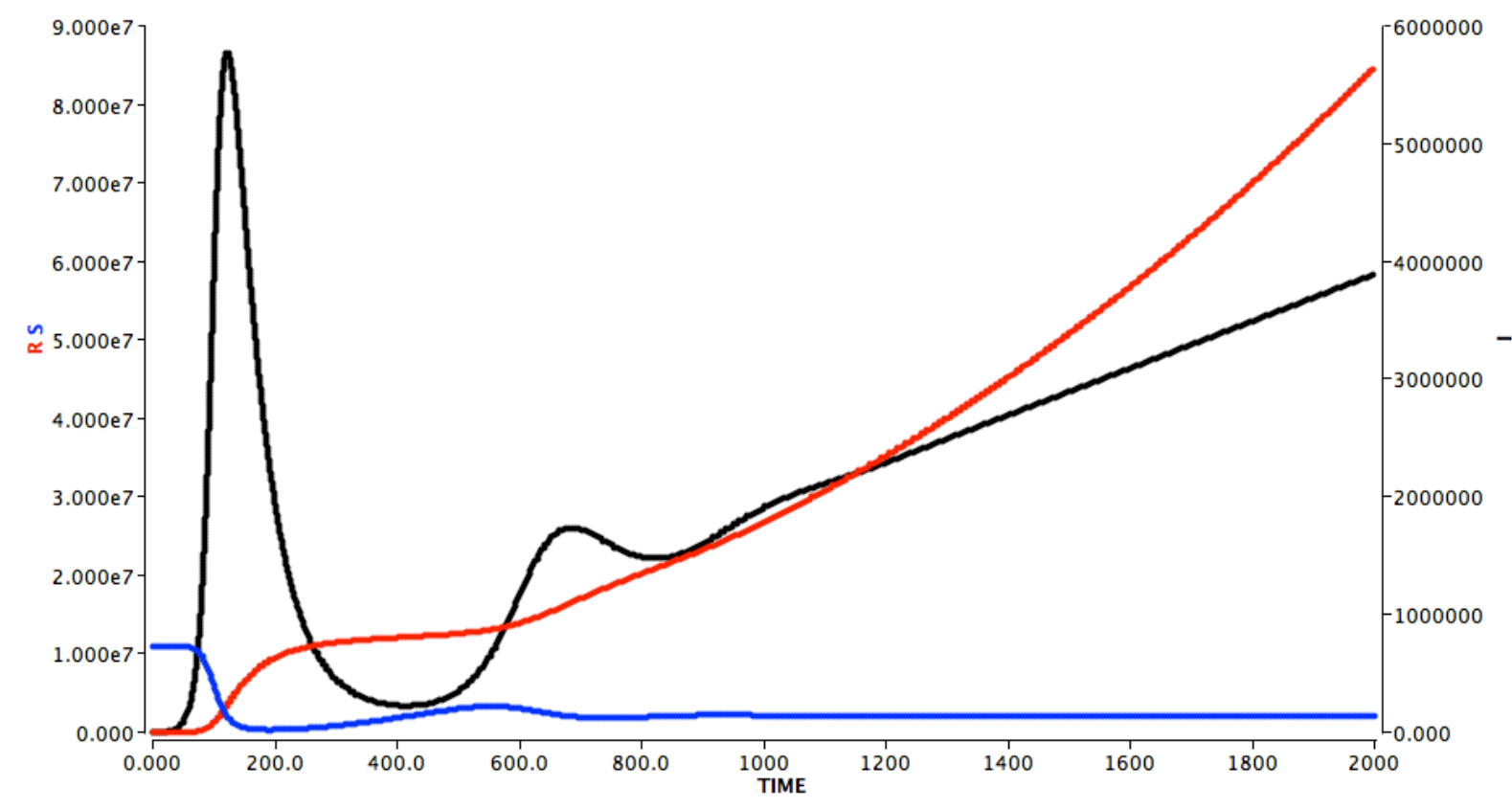

Figure 2. A simulation of the basic two-nation model showing how the density of infected people ( $I$, in black) rises, decreases and then increases again. The density of susceptible people ( $S$, in blue) declines initially causing the infection to start declining. It then starts increasing due to influx of soldiers and refugees, causing the rebound in infections. The density of people who recover ( $R$, in red) increases throughout the process.

We only show representative plots to demonstrate this case. We note that this model is not specific to any disease nor do we estimate model parameters specific to any pathogen.

Our objective is only to demonstrate that such a situation is indeed feasible. It would be possible to fit these mathematical models to data, should adequate data become available.

\section{AN EPIDEMIC CAN BE DECREASED DURING TIMES OF CONFLICT}

Epidemics could be reduced for some time during conflicts. This could happen due to:

a) mobility of people being reduced. For example, lower incidence of HIV has been reported in Angola and is attributed to reduced mobility due to conflicts [1].

b) increase in susceptible population during conflict due to increase in migration.

We show this effect can be simulated by an increase in the value of $S(t)$ after some time. The increase $(X)$ is modelled as a piecewise linear function: it is 0 before some time and increases linearly after some time. The model is shown below.

$$
\begin{aligned}
& \frac{\mathrm{d} S}{\mathrm{~d} t}=-\beta I S+\alpha X, \\
& \frac{\mathrm{d} I}{\mathrm{~d} t}=\beta I S-v I, \\
& \frac{\mathrm{d} R}{\mathrm{~d} t}=v I . \\
& X=\left\{\begin{array}{cc}
0, & t<t_{i} \\
\delta \cdot\left(t-t_{i}\right), & t \geq t_{i} .
\end{array} .\right.
\end{aligned}
$$

A simulation of this outcome is shown in Figure 3 and it can be seen that the density of infections declines (after 100 time units) and then comes back up again. 


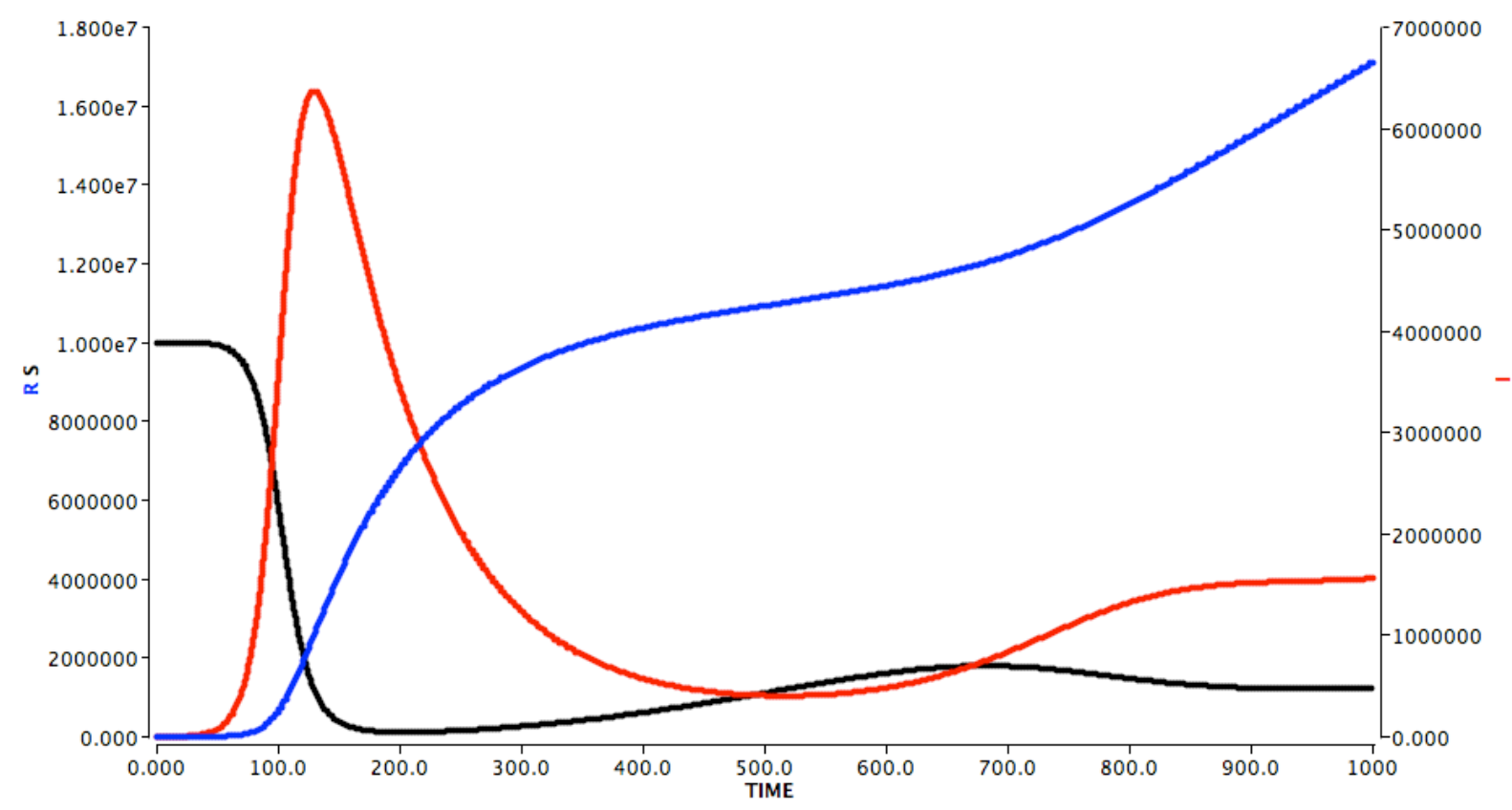

Figure 3. A simulation of the migration model showing how the density of infected people ( $I$, in red) rises and then decreases. The density of susceptible people ( $S$, in blue) declines initially causing the infection to start declining. It then starts increasing due to migration, causing the rebound in infections. The density of people who recover $(R$, in red) increases throughout the process.

\section{EPIDEMICS COULD REAPPEAR OR BE DIMINISHED AFTER THE END OF CONFLICTS}

Epidemics could reappear after the end of conflicts due to migration of refugees displaced during the conflict. This highlights the need for sustained humanitarian aid missions even after conflicts have ceased.

This effect can be simulated by an increase in $S(t)$. The model has been introduced in the previous section. Figure 3 shows that the infection can decline over certain periods of time; in the simulation it declines between 100 and 500 time units, before rebounding again.

After a conflict ends, there could also be migration of refugees and peacekeepers, all of whom could either add to the susceptible or infected pool. This could cause a resurgence of the epidemic. We show another plausible model for this below. This model has an influx (after a certain time) in the susceptible $(S)$ and infected $(I)$ compartments. The increase is modelled as a piecewise linear function.

$$
\begin{aligned}
& \frac{\mathrm{d} S}{\mathrm{~d} t}=-\beta I S+\alpha_{1} X_{1}, \\
& \frac{\mathrm{d} I}{\mathrm{~d} t}=\beta I S-v I+\alpha_{2} X_{2}, \\
& \frac{\mathrm{d} R}{\mathrm{~d} t}=v I \text {. } \\
& X_{1}=\left\{\begin{array}{cc}
0, & t<t_{i}, \\
\delta_{1} \cdot\left(t-t_{i}\right), & t \geq t_{i} .
\end{array}\right. \\
& X_{2}=\left\{\begin{array}{cc}
0, & t<t_{i}, \\
\delta_{2} \cdot\left(t-t_{i}\right), & t \geq t_{i} .
\end{array}\right.
\end{aligned}
$$

Results of a simulation are shown in Figure 4. The plot shows how the density of infected people rises, then decreases and then increases again. 


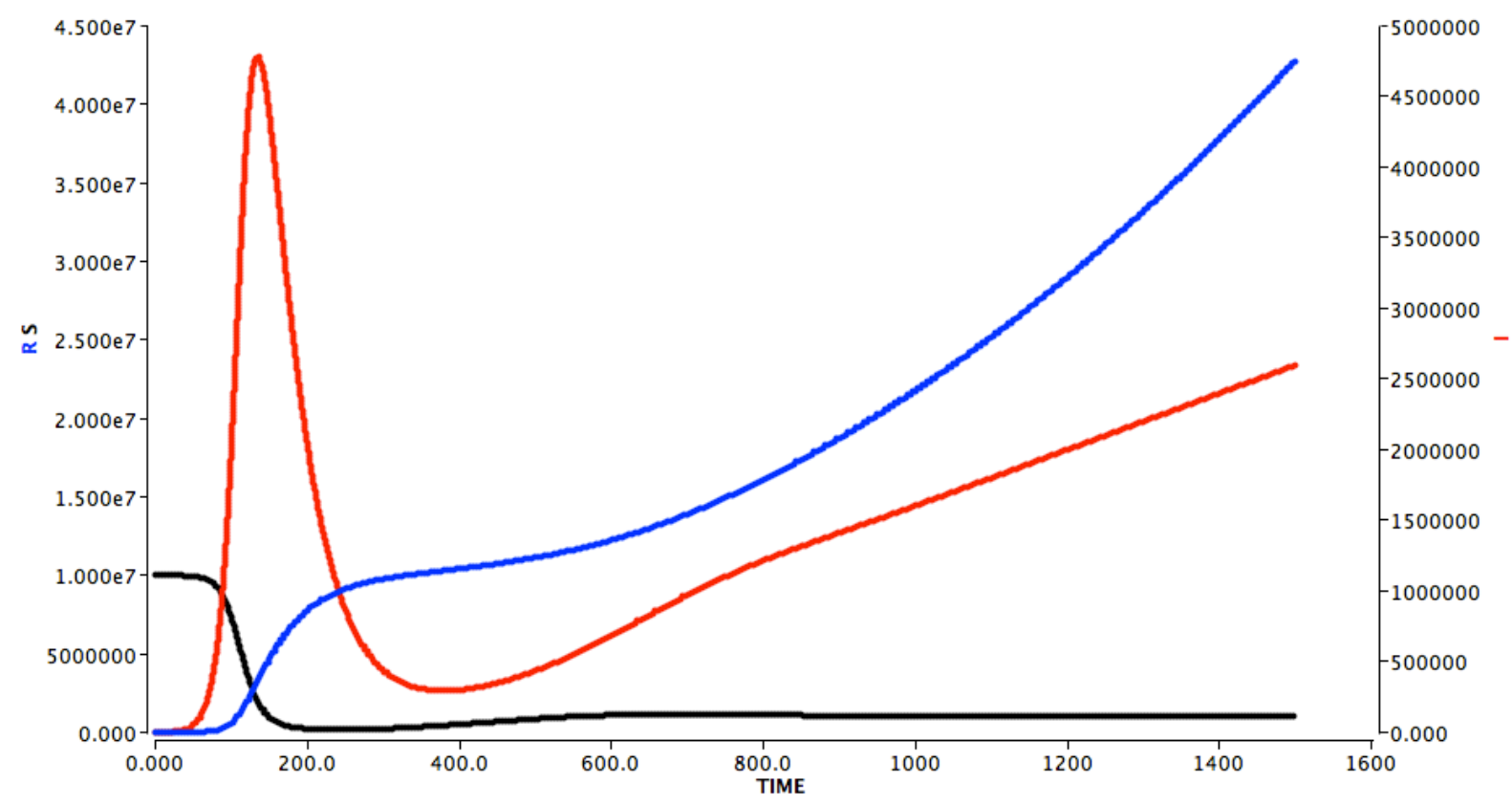

Figure 4. A simulation of a model after a conflict with migration of refugees and peacekeepers in both the infected and susceptible populations. The simulation shows how the density of infected people ( $I$, in red) rises, then decreases and then increases again. The density of susceptible people ( $S$, in black) declines and then increases again after a conflict (due to migration of refugees and peacekeepers). The density of people who recover $(R$, in blue) increases throughout the duration of the simulation.

\section{THE EFFECTS OF MIGRATION ON DISEASES}

As the previous cases demonstrate, migration has a significant effect on disease spread, during and after conflicts. Here we examine in more detail the different effects migration can have on spread of infectious diseases. Other additional factors that can compound recovery from epidemics, caused in part by both migration and conflicts, are:

a) breakdown of medical infrastructure,

b) over-crowding, and,

c) unsanitary facilities.

Most of these would affect the rate of recovery (parameter $v$ ) in the model. We can simulate these effects by lowering the value of $v$ in the model. The rate of recovery $(v)$ is at baseline before some time and then is assumed to decrease linearly with time. We can look at other functional forms, but this is a very basic and simple formulation. In the future we can fit more complex functions, when data becomes available.

The model is as follows:

$$
\begin{aligned}
& \frac{\mathrm{d} S}{\mathrm{~d} t}=-\beta I S, \\
& \frac{\mathrm{d} I}{\mathrm{~d} t}=\beta I S-v I, \\
& \frac{\mathrm{d} R}{\mathrm{~d} t}=v I . \\
& v=\left\{\begin{array}{cc}
v_{0}, & t<t_{i} . \\
v_{0}-\eta \cdot\left(t-t_{i}\right), & t \geq t_{i} .
\end{array} .\right.
\end{aligned}
$$

A simulation of this outcome is shown in Figure 5. 


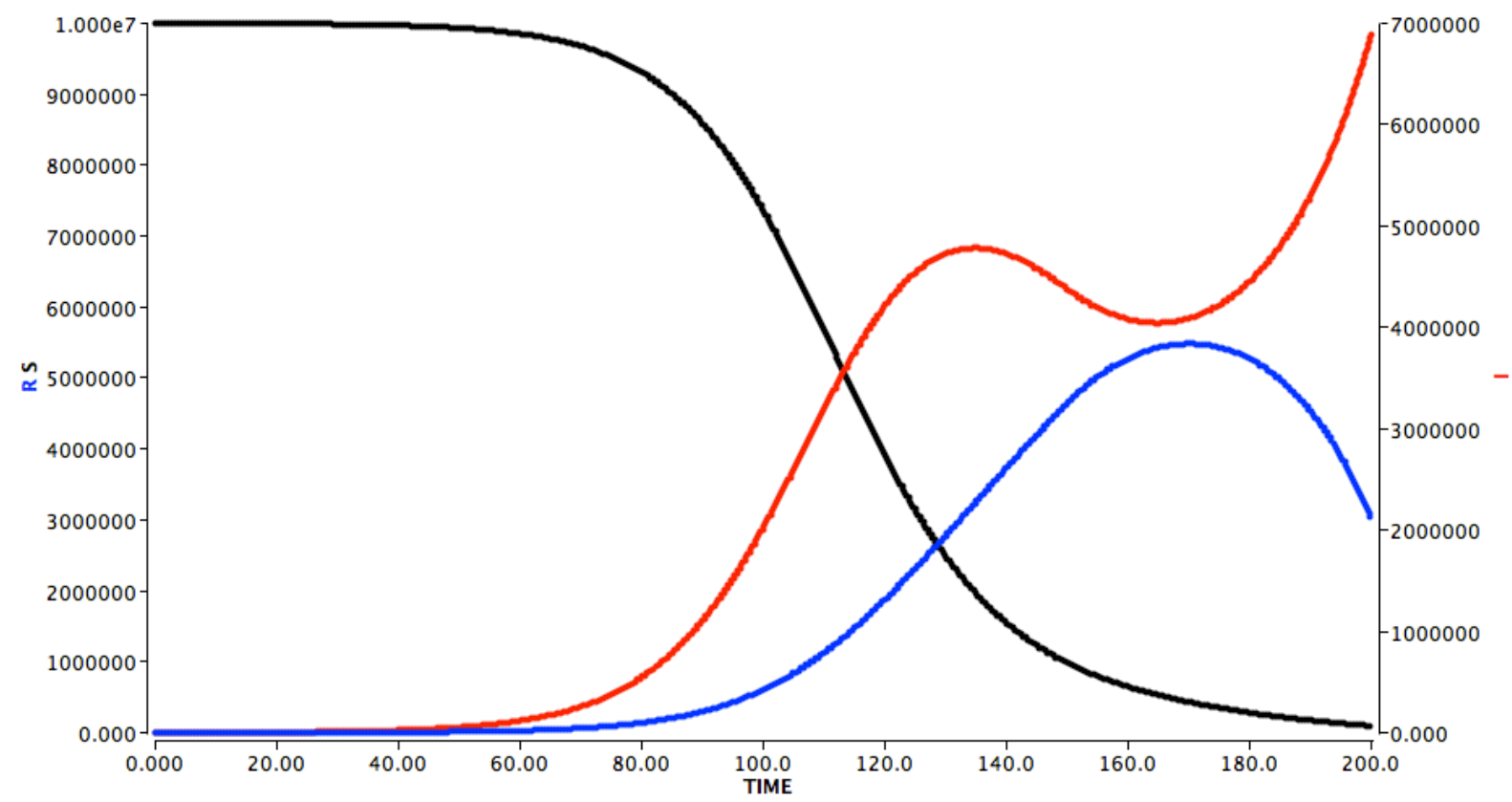

Figure 5. A simulation of the migration model with additional factors showing how the density of infected people ( $I$, in red) rises, then decreases and then increases again. The density of susceptible people ( $S$, in black) declines throughout this process. The density of people who recover $(R$, in blue) increases and then decreases again due to the decline in the rate of recovery (due to breakdown in infrastructure).

Migration has a significant effect on infectious diseases, both during and after conflicts. It may make more people susceptible to diseases, or isolate people from diseases in regions with poor connectivity. In future work, we will look at spatial models that couple migration, disease and conflicts.

\section{SCENARIO WITH MULTIPLE FACTORS}

We acknowledge the complexity of looking at conflict, disease and socio-economics as a coupled system. Many of the factors that we outlined above may co-occur with each other. As an example, we present one such integrated model below. The model below incorporates an increase in the susceptible population $S(t)$. It also simulates a breakdown of critical infrastructure that causes a decline in the recovery rate $(v)$.

$$
\begin{aligned}
& \frac{\mathrm{d} S}{\mathrm{~d} t}=-\beta I S, \\
& \frac{\mathrm{d} I}{\mathrm{~d} t}=\beta I S-v I, \\
& \frac{\mathrm{d} R}{\mathrm{~d} t}=v I . \\
& v=\left\{\begin{array}{cc}
v_{0}, & t<t_{i}, \\
v_{0}-\eta \cdot\left(t-t_{i}\right), & t \geq t_{i} .
\end{array} .\right. \\
& X=\left\{\begin{array}{cc}
0, & t<t_{i}, \\
\delta \cdot\left(t-t_{i}\right), & t \geq t_{i} .
\end{array} .\right.
\end{aligned}
$$

\section{MODELLING INTERVENTIONS}

If there is available data, future modellers can even try to estimate the increase in recovery rate $(v)$ required to reduce epidemics below a certain threshold. This assumes that the intervention can only effect $v$. Other interventions can affect the susceptible $(S)$ and infected (I) populations by targeted vaccinations. 
Such a model is giveni in this section. Currently, there are no data to calibrate these models. It is hope that emerging technologies like smartphones in developing nations and remote sensing by satellites can enable modellers to get some approximate estimates of model parameters, like populations of infected and susceptible people [5]. This may enable forecasts of amount of humanitarian aid required to reduce an infection below a threshold. We model an intervention as an increase (piecewise linear) in the rate of recovery of infected individuals $v$.

$$
\begin{aligned}
& \frac{\mathrm{d} S}{\mathrm{~d} t}=-\beta I S, \\
& \frac{\mathrm{d} I}{\mathrm{~d} t}=\beta I S-v I, \\
& \frac{\mathrm{d} R}{\mathrm{~d} t}=v I . \\
& v=\left\{\begin{array}{cc}
v_{0}, & t<t_{i}, \\
v_{0}+\eta \cdot\left(t-t_{i}\right), & t \geq t_{i} .
\end{array} .\right. \\
& X=\left\{\begin{array}{cc}
0, & t<t_{i}, \\
\delta \cdot\left(t-t_{i}\right), & t \geq t_{i} .
\end{array}\right.
\end{aligned}
$$

First, we show the simulations from a model without the intervention, Figure 6.

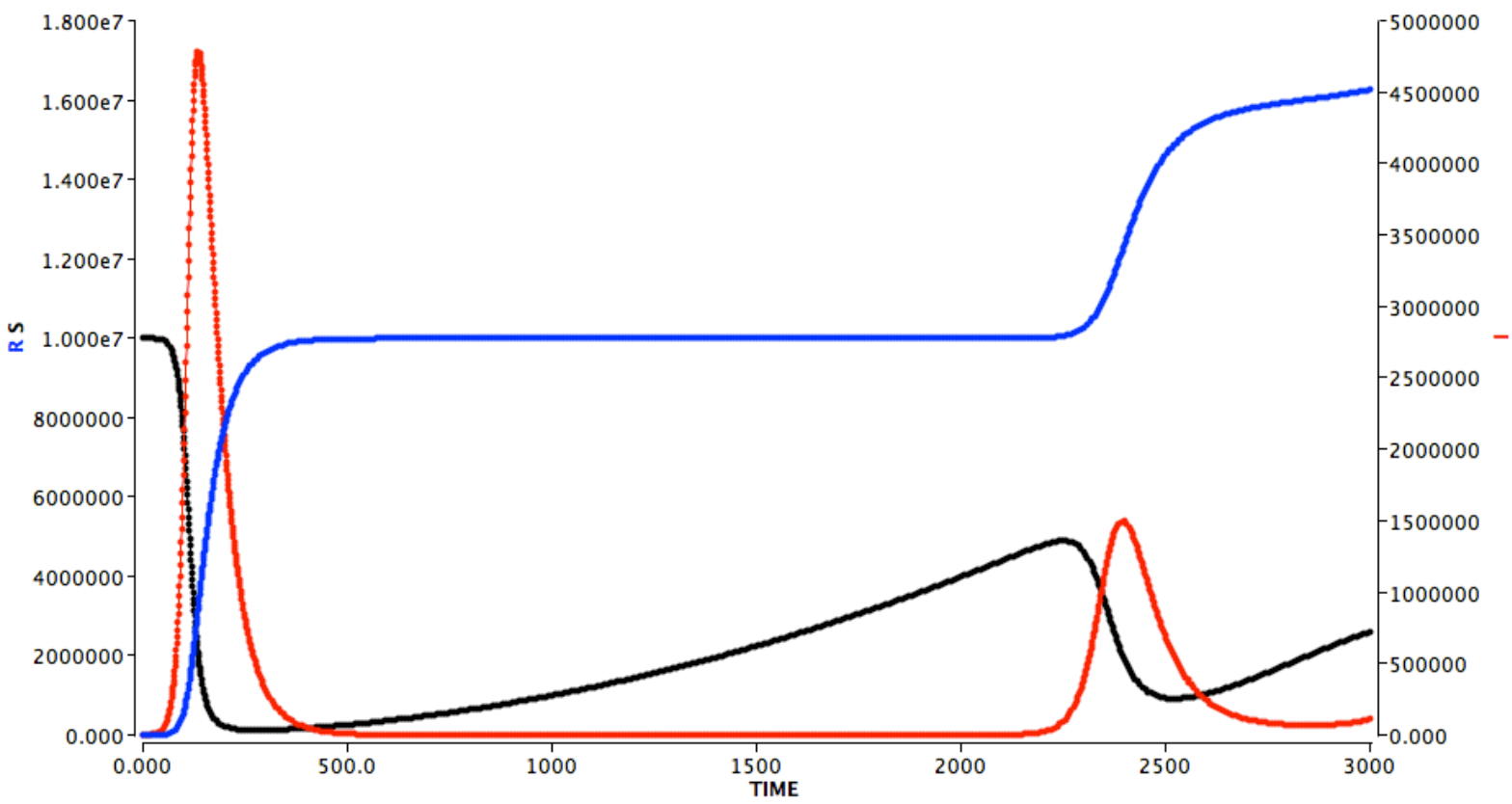

Figure 6. A simulation of the migration model showing how the density of infected people ( $I$, in red) rises, then decreases and then increases again (due to migration). The density of susceptible people ( $S$, in black) declines and then increases due to migration.

We then show the effect of an intervention of increasing the rate of recovery $(v)$ by improved access to health services and vaccination, Figure 7). The intervention, initiated after some time, has the effect of checking the rebound in infections seen in the model without interventions.

\section{EFFECT OF POVERTY ON DISEASES}

We can simulate the effect of poverty on disease propagation by coupled models of socio-economics and diseases. The gross domestic product (GDP) of a country may affect the rate at which infected patients recover (parameter $v$ ). Poorer nations may have a lower value of $v$ thereby compromising their chances of recovering from epidemics after a conflict. We parameterize $v$ as a function of GDP. 


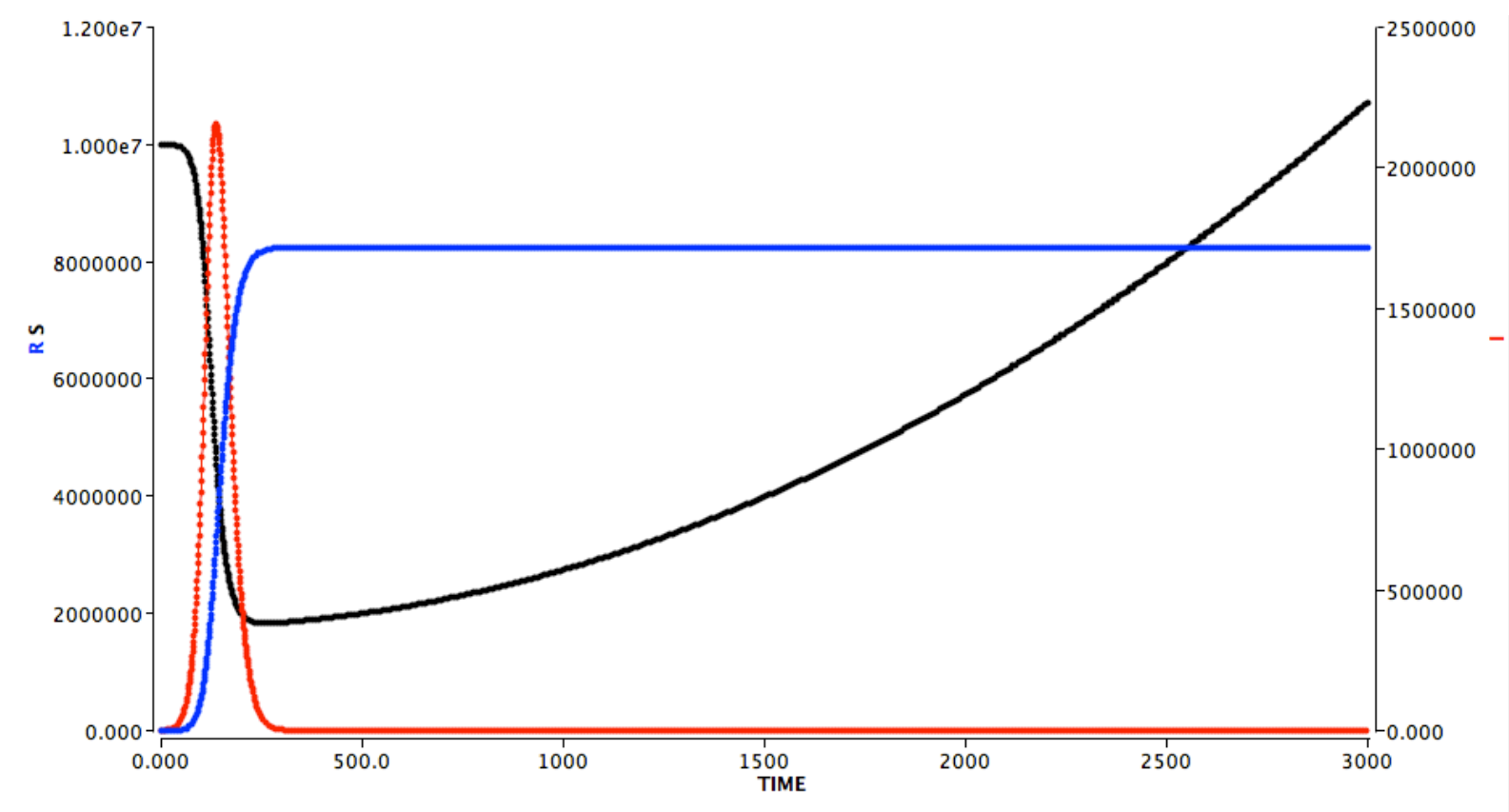

Figure 7. A simulation of the migration model with an intervention of increasing the rate of recovery after some time $(v)$. This has the effect of checking the increase in infected people seen in the model without intervention. The density of infected people ( $I$, in red) rises and then decreases.

The corresponding model is as follows:

$$
\begin{gathered}
\frac{\mathrm{d} S}{\mathrm{~d} t}=-\beta I S, \\
\frac{\mathrm{d} I}{\mathrm{~d} t}=\beta I S-v I, \\
\frac{\mathrm{d} R}{\mathrm{~d} t}=v I . \\
f(G D P), \\
v=\left\{\begin{array}{cc}
t<t_{i} . \\
f(G D P)-\eta(G D P) \cdot\left(t-t_{i}\right), & t \geq t_{i} .
\end{array}\right.
\end{gathered}
$$

\section{SOCIO-ECONOMICS OF DISEASE SPREAD}

Conflicts can contribute to and also may be caused by diseases [6]. Here we consider the socio-economics of infectious disease spread.

Diseases can breakdown resources, reduce GDP, and deplete resources. This may in some instances compel these nations to initiate conflicts for acquiring resources externally [6].

We look at coupled models of socio-economics and disease spread. We assume that the rate of recovery $(v)$ from infections is related to the GDP.

The model is shown further in the text. We assume there are two countries that are competing with each other economically. Their GDPs are denoted by $x$ and $y$. They each have epidemics where the rate of recovery $(v)$ is dependent on GDP.

$$
\begin{gathered}
\frac{\mathrm{d} x}{\mathrm{~d} t}=\alpha x-\sigma x y, \\
\frac{\mathrm{d} y}{\mathrm{~d} t}=-\delta y+\gamma x y, \\
\frac{\mathrm{d} S_{j}}{\mathrm{~d} t}=-\beta I_{j} S_{j},
\end{gathered}
$$




$$
\begin{gathered}
\frac{\mathrm{d} I_{j}}{\mathrm{~d} t}=\beta I_{j} S_{j}-v_{j} I_{j}, \\
\frac{\mathrm{d} R_{j}}{\mathrm{~d} t}=v_{j} I_{j}, \\
v_{1}=f(y), v_{2}=f(x) .
\end{gathered}
$$

with $j=1,2$ in (42)-(44). This is a very simple model where two countries that are competing economically have GDPs $x$ and $y$. The rate of recovery $(v)$ is dependent on GDP through a function $f(G D P)$.

In this model, the GDP fluctuates over time due to competition between the two countries (as in a predator-prey model). The rate of recovery from infections also changes due to linked dynamics with socio-economics (GDP).

Formulated demonstrate the vicious cycle of poverty, disease and conflicts especially in some developing nations. This also suggests that aid organizations should take an integrated public health approach that is combined with efforts to aid socio-economic development.

We also show another model where we model the public health burden of epidemics.

$$
\begin{gathered}
\frac{\mathrm{d} x}{\mathrm{~d} t}=\alpha x-\sigma x y, \\
\frac{\mathrm{d} y}{\mathrm{~d} t}=-\delta y+\gamma x y, \\
\frac{\mathrm{d} S_{j}}{\mathrm{~d} t}=-\beta I_{j} S_{j}, \\
\frac{\mathrm{d} I_{j}}{\mathrm{~d} t}=\beta I_{j} S_{j}-v_{j} I_{j}, \\
\frac{\mathrm{d} R_{j}}{\mathrm{~d} t}=v_{j} I_{j}, \\
v_{1}=f(y), v_{2}=f(x) .
\end{gathered}
$$

Again we assume a scenario where two countries are competing economically and have GDPs $x$ and $y$. As before the rate of recovery $(v)$ is dependent on GDP through a function $f(G D P)$. Additionally, the GDPs of the countries are now also reduced based on a function $(g)$ of the number of infected people. This models the economic impact of epidemics.

\section{A SCENARIO OF A COMPLEX INTERACTION OF DISEASE SPREAD IN A PREDATOR-PREY SYSTEM}

Our models are general enough to capture conflict-disease dynamics in other species. We consider a final scenario which may occur in other species. Consider two species, one of which preys on the other (predator-prey system). Assume that there is an infectious disease that infects only one species (say, the prey).

The model is as follows:

$$
\begin{gathered}
\frac{\mathrm{d} x}{\mathrm{~d} t}=\alpha x-\sigma x y, \\
\frac{\mathrm{d} y}{\mathrm{~d} t}=-\delta y+\gamma x y, \\
Y=S+I+R, \\
\frac{\mathrm{d} S}{\mathrm{~d} t}=-\beta I S, \\
\frac{\mathrm{d} I}{\mathrm{~d} t}=\beta I S-v I, \\
\frac{\mathrm{d} R}{\mathrm{~d} t}=v I,
\end{gathered}
$$

Here we have a predatory-prey Lotka-Volterra model coupled to an SIR model. Whenever the density of predator $(x)$ goes down, the prey $(y)$ goes up. The total population of the prey 
$(y)$ is composed of $S+I+R$. Some fraction of $S$ is going to increase whenever the population of prey increases.

From the discussion in the previous sections, when we increase the susceptible population, we can get oscillations of infections going up and down. We hypothesize that in certain cases the pathogen may evolve to reproduce around the peaks of the prey population.

\section{PRELIMINARY TRENDS}

We acknowledge there is very scarce data on conflicts and diseases simultaneously with good resolution and fidelity. Nevertheless we would like to point out some trends from whatever data sources do exist.

We add the caveat that there is very scarce data and the reliability and granularity with which this data was collected also needs to be critically examined.

In the future, it may be possible to capture data at higher granularity and build data driven mathematical models.

We show some trends using gapminder.org, open data and visualization platform. Figure 8 shows a visualization of deaths in battles (per 100000 people) vs. deaths due to malaria. Shown are trends (no statistical significance or causation is implied) for three countries from 2002 to 2004 . The visualization is freely available online.

For Angola, which had been suffering a war from 1975 to 2002, we observe that deaths in battles decreased over this timespan, whereas deaths due to malaria increased and then decreased.

The Democratic Republic of Congo (DRC) had been suffering from a war from 1998 to 2003. There is still an ongoing conflict with terrible casualties till date. For the DRC, we observe that battle deaths decrease over time and deaths due to malaria unfortunately show an increasing trend.

Unfortunately, Burundi has seen a steady increase in battle deaths with no visually appreciable trend in deaths due to malaria.

We would like to reiterate that we do not imply statistical significance or causality. The data sources and collection methods need to be critically examined. Our objective is only to highlight trends and make a case for a more concerted effort for data collection in conflict prone regions.

We also look at the number of refugees over time for three countries (Iran, Pakistan and Afghanistan) in a region that has seen conflicts for the last few decades, Figure 9.

We also look at health spending as a percentage of GDP for some countries, see Figure 10. We observe that for Burundi there was a small trend of increased spending on health whereas for Syria there has been a small decline. In the future, if there is reliable data, it may be possible to estimate the rate of recovery in SIR models (parameter $n$ ) using these kinds of datasets.

Overall, we find a disturbing trend of a global increase in the number of refugees over the last two decades [7]. This puts a large fraction of the world population at risk. This is also underscored by the current conflict and Ebola epidemic in the Democratic Republic of Congo as of 2019.

\section{DISCUSSION}

The effects of conflict on infectious diseases are multi-faceted and complex. There have been observations of both increase and decrease in disease outbreaks during conflicts. Epidemics have been observed to be both initiated and extinguished by conflicts [1]. However there is no unified mathematical model that explains all these counter-intuitive observations. 


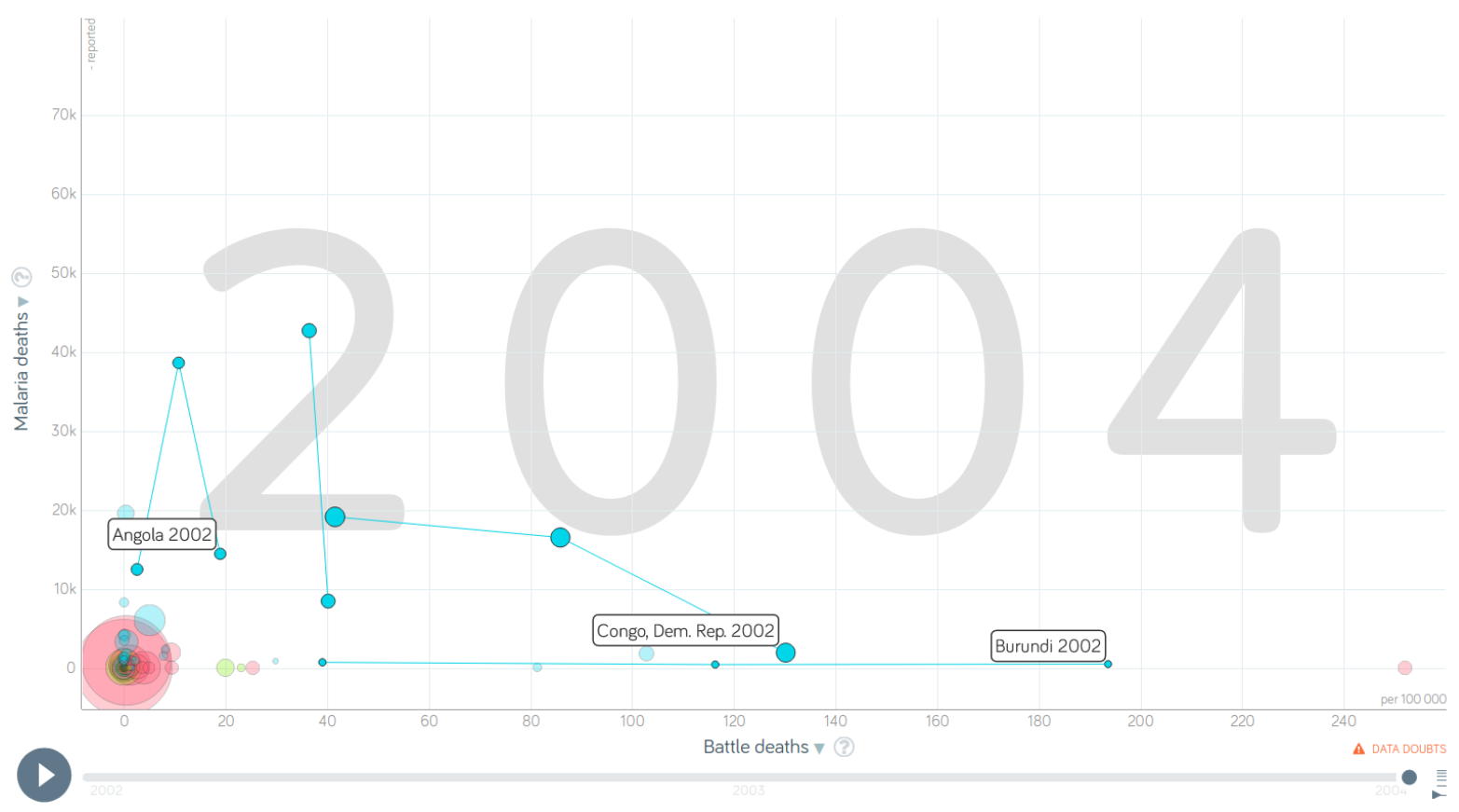

Figure 8. A visualization of deaths in battles vs. deaths due to malaria. The lines show a trend over time from 2002 to 2004 for three countries. The countries shown are Angola, Democratic Republic of Congo and Burundi. Based on free material from gapminder.org, CC-BY license. The visualization can be viewed online'.

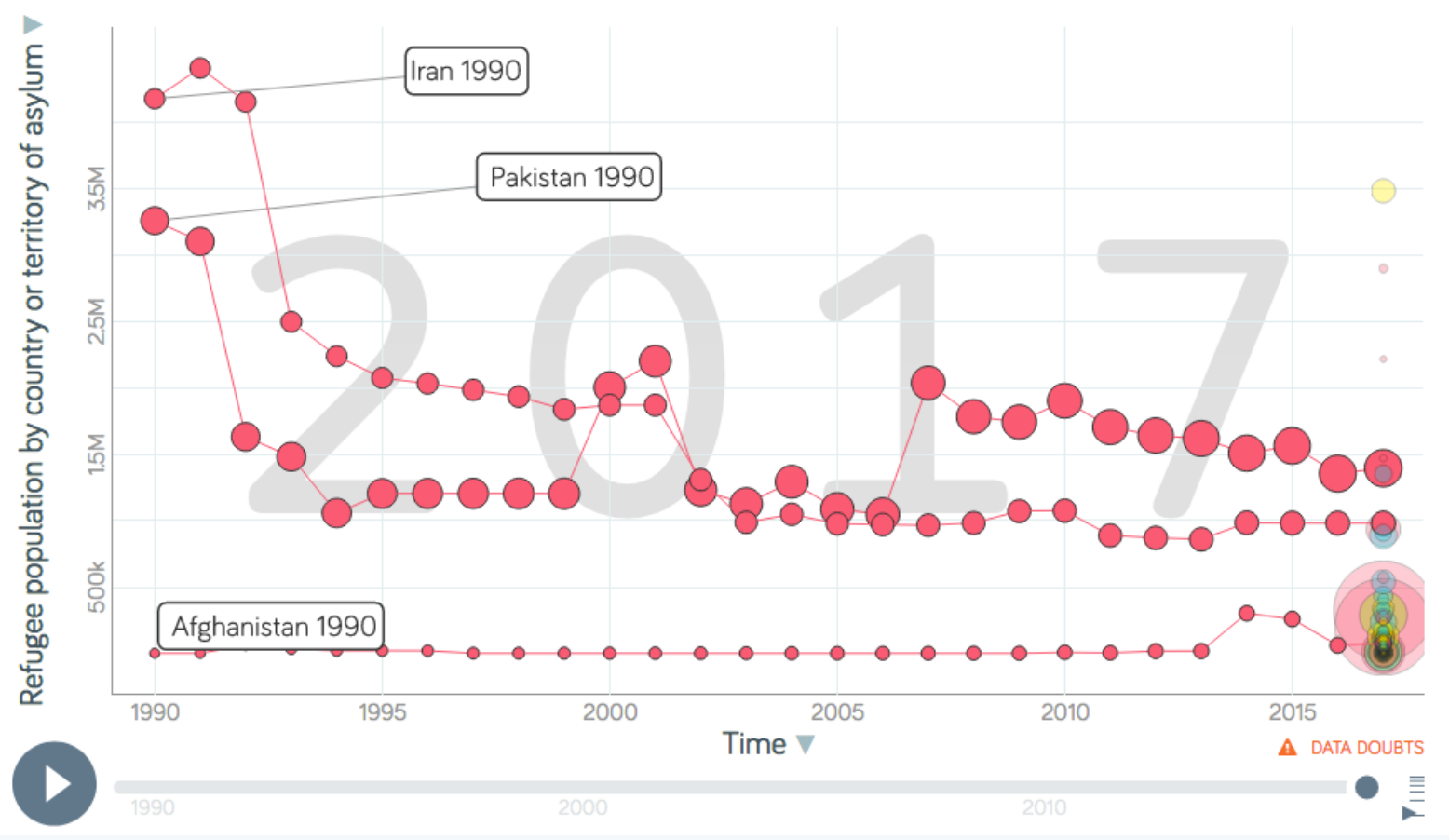

Figure 9. A visualization of the number of refugees in Iran, Pakistan and Afghanistan over time. The lines show a trend over time from 1990 to 2017 for these three countries. Based on free material from gapminder.org, CC-BY license. The visualization can be viewed online ${ }^{2}$. 


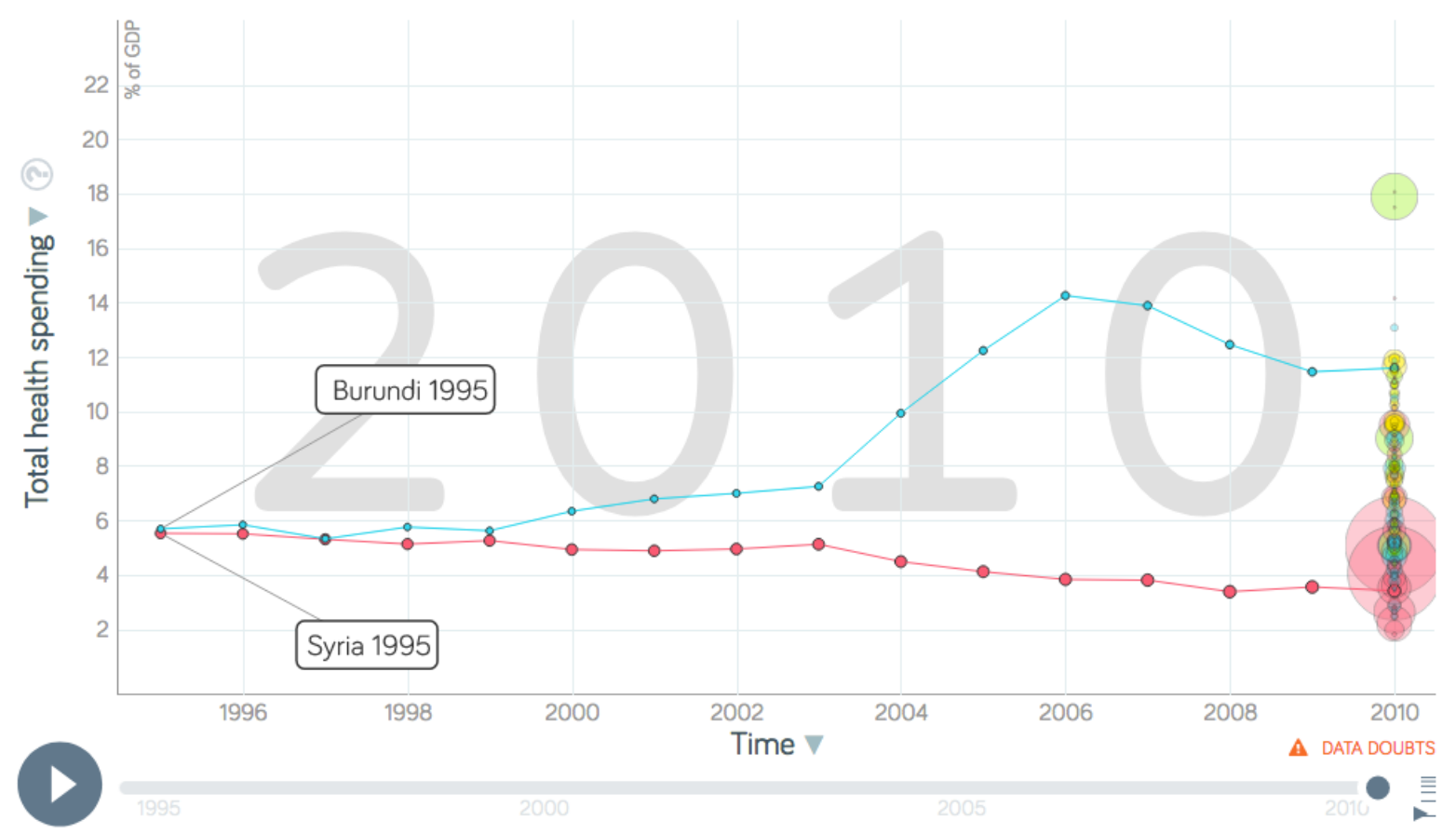

Figure 10. A visualization of the health spending as a percentage of GDP in Burundi and Syria over time. The lines show a trend over time from 1995 to 2010 for these three countries. Based on free material from gapminder.org, CC-BY license. The visualization can be viewed online ${ }^{3}$.

The recent appearance of emerging pathogens like Zika and Ebola virus in conflict-prone regions, highlights the need for a quantitative framework that integrates both diseases and conflicts.

Such models can be a first step towards policy, spreading public awareness and may also be a tool for public health professionals in conflict zones. Quantitative techniques like these may also help us predict possible emerging hotspots for emerging diseases [8].

In this work, we introduce a set of mathematical models to understand the role of conflicts in epidemics. Our mathematical framework has the potential to explain counterintuitive observations and the complex role of human conflicts in epidemics.

\section{THE ROLE OF NON-LINEAR MODELS IN UNDERSTANDING COMPLEX SYSTEMS}

We suggest that non-linear mathematical models can help us understand why conflicts may both increase and decrease epidemics. We outline a few situations which explain the previously counter-intuitive observations. We develop coupled non-linear models of conflict, socio-economics and disease spread.

We show representative plots to demonstrate the role of dynamical systems in this field.

We note that the models are not specific to any disease nor do we estimate model parameters specific to any pathogen. Our objective is only to demonstrate that such a situation is indeed feasible. It should be possible to fit similar mathematical models to data, once such data becomes available. We note that the type of disease also matters. For example, a vector-borne disease will have different characteristics compared to sexually transmitted diseases. Hence diseases may have different effects on conflicts based on their type and mode of transmission.

Conflicts also cause a higher incidence of stress and trauma related diseases like diabetes and strokes [9]. 
Conflicts can also help introduce new pathogens. Invading armies can introduce new diseases to a population that has no immunity (the English and the American armies introduced smallpox to Native Americans) or the invading army itself may be exposed to a novel pathogen (Napoleon's army suffered from typhus during the Russian invasion) [10]. We speculate that diseases may, in certain cases, even pause conflicts.

\section{POLICY IMPLICATIONS}

At the end of conflicts, epidemics could go away or reappear due to migration of aid workers, displaced populace, etc. Timely humanitarian intervention is key to reducing the spread of diseases.

The policy implications are that public health officials will need to work closely with peacekeeping missions and humanitarian aid workers to manage crises, both during and after conflicts.

Migration has a significant effect on infectious diseases during and after conflicts. This suggests that steps taken to manage refugee crises during and after conflicts are critical in preventing outbreaks of infectious diseases.

Our models demonstrate the vicious cycle of poverty, disease and conflicts, especially in some developing nations. Diseases can cause more poverty due to the increased public health burden. Ultimately this may also lead to conditions that encourage conflicts for resources.

This suggests that aid and peacekeeping organizations should take an integrated approach that combines:

1) public health measures,

2) efforts to aid socio-economic development,

3) peacekeeping in the region.

We also suggest that managing public health crises and reducing poverty can have significant knock-on effects including, we hope optimistically, reduction of conflicts. It is intriguing to speculate that perhaps an integrated approach where public health intervention is coupled with nation-building efforts, for example to build technological infrastructure and international scientific collaboration networks, may help these countries recover in the long term [11].

We do however acknowledge the difficulties that humanitarian organizations will face in reaching communities during times of conflict.

\section{FUTURE WORK}

There are certain aspects of this complex system that we have not incorporated which may form the subject of future investigations. We outline some of those factors here. Human trafficking during and after conflicts is an unfortunately common occurrence in conflict zones. The trauma it leaves behind in victims alone is unfathomable. Humanitarian aid or peacekeeping organizations also sometimes unfortunately exploit people in conflict zones, as has happened in the Yugoslavia in the 1990s and more recently in Haiti. These incidents inflict further trauma on the victims. They also erode trust in these organizations and further delay and complicate recovery efforts after conflicts.

Another area of future investigation will be the humanitarian and ethical aspects and other benefits that come from welcoming refugees and displaced people. Refugees can bring in a lot of diversity into another country and enrich it in many ways [8]. We can investigate how foreign aid and influx of refugees can help these countries recover after conflicts. 


\section{CONCLUSION}

This work is a step towards a quantitative model of disease spread in conflicts. Our model explains apparently inconsistent observations on disease spread during conflicts. A multitude of possibilities are explained in a quantitative framework. Our work also highlights the importance of simple mathematical models and the perils of applying linear thinking to nonlinear complex systems. Non-linear models produce counter-intuitive results; disease spread is a non-linear phenomenon which produces counter-intuitive results. Such mathematical models have been used in the past to model diverse complex systems ranging from socioeconomic to biological systems [2]. We note that similar mathematical models have also been used to explain crime and violence in human societies [8].

Our work raises the hope for a predictive model that may be of use to first responders and public health officials in conflict hotspots. Mathematical models of joint epidemic and conflict risk would be of considerable interest to future humanitarian and peacekeeping missions.

\section{ACKNOWLEDGEMENTS}

This work is dedicated to Dadu. This is also dedicated to the millions of people around the world who are affected by conflicts, diseases, forced migration and human trafficking.

\section{REMARKS}

${ }^{1}$ https://www.gapminder.org/tools/\#\$state\$time \$value=2004\&delay:1200;\&marker\$select@\$ country $=$ ago $\&$ trail StartTime $=2002 ; \& \$$ country $=$ cod $\&$ trail StartTime $=2002 ; \& \$$ country $=$ uga $\& \mathrm{t}$

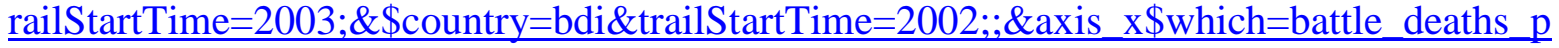
er_100_000\&domainMin:null\&domainMax:null\&zoomedMin:null\&zoomedMax:null\&scale Type=linear\&spaceRef:null;\&axis_y\$which=malaria_number_of_deaths_reported\&domain Min:null\&domainMax:null\&zoomedMin:null\&zoomedMax:null\&spaceRef:null;;;\&charttyp $\mathrm{e}=$ bubbles.

${ }^{2}$ https://www.gapminder.org/tools/\#\$state\$time\$value=2017\&delay:1179;\&marker\$select@\$ country=afg \& trailStartTime=1990; \& $\$$ country=pak\&trailStartTime=1990\&labelOffset $@: 0.3$ 82\&:0.051;;\& \$country=irn\&trailStartTime=1990\&labelOffset@ $: 0.28 \&: 0.027 ; ; ; \&$ axis $\mathrm{x} \$$ wh ich=time\&domainMin:null\&domainMax:null\&zoomedMin:null\&zoomedMax:null\&scaleTy pe=time\&spaceRef:null;\&axis_y $\$$ data=data_wdi\&which=sm_pop_refg\&domainMin:null\&d omainMax:null\&zoomedMin:null\&zoomedMax:null\&spaceRef:null;;;\&chart-type=bubbles.

${ }^{3}$ https://www.gapminder.org/tools/\#\$state\$time\$value=2010\&delay:1179;\&marker\$select@\$ country=bdi\&trailStartTime=1995\&labelOffset@ :0.249\&:-

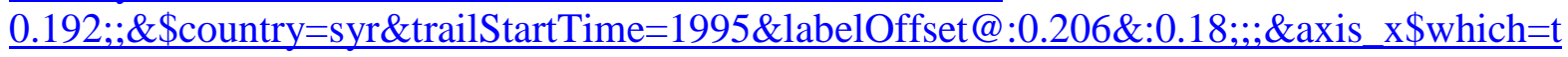
ime\&domainMin:null\&domainMax:null\&zoomedMin:null\&zoomedMax:null\&scaleType=ti me\&spaceRef:null;\&axis_y \$which=total_health_spending_percent_of_gdp\&domainMin:nul 1\&domainMax:null\&zoomedMin:null\&zoomedMax:null\&spaceRef:null;;;\&charttype $=$ bubbles.

\section{REFERENCES}

[1] McInnes, C.: Conflict, HIV and AIDS: A new dynamic in warfare?

Global Change, Peace \& Security 21(1), 99-114, 2009,

http://dx.doi.org/10.1080/14781150802659440,

[2] Banerjee, S.; Perelson, A.S. and Moses, M.: Modelling the effects of phylogeny and body size on within-host pathogen replication and immune response.

Journal of The Royal Society Interface 14(136), 20170479, 2017,

http://dx.doi.org/10.1098/RSIF.2017.0479,

[3] Macey, R.I. and Oster, G.F.: Berkeley Madonna. Version 8.0.

University of California at Berkeley, Berkeley, 2001, 
[4] Banerjee, S.: repository_conflict_diseases. https://bitbucket.org/neelsoumya/repository_conflict_diseases, accessed April 2019,

[5] World Health Organization: Refugee population by country or territory of asylum. https://www.who.int/globalchange/publications/oeh0401/en/index6.html, accessed April 2019,

[6] Altman, D.: Causal Effects of Epidemics on Conflict: A Summary of the Evidence. SemanticScholar report, 2010,

[7] Worldbank: Refugee population by country or territory of asylum. https://data.worldbank.org/indicator/sm.pop.refg, accessed July 2019,

[8] Banerjee, S.: An Immune System Inspired Theory for Crime and Violence in Cities. Interdisciplinary Description of Complex Systems 15(2), 133-143, 2017, http://dx.doi.org/10.7906/indecs.15.2.2,

[9] Maxmen, A.: Violence and diabetes boom in the Middle East. Nature, 4 August 2017, http://dx.doi.org/10.1038/nature.2017.22371,

[10] Roy, K. and Ray, S.: War and epidemics: A chronicle of infectious diseases. Journal of Marine Medical Society 20(1), 50-54, 2018, http://dx.doi.org/10.4103/jmms.jmms_34_18,

[11]Banerjee, S.: Analysis of a Planetary Scale Scientific Collaboration Dataset Reveals Novel Patterns.

In: Complex Systems Digital Campus-15 - World e-Conference. Complex Systems Digital Campus, 2015. 